\title{
Potential electron acceptors for anaerobic methane oxidation during long-term incubations of lake sediments
}

\author{
HANNI VIGDEROVICH ${ }^{1}$, WERNER ECKERT ${ }^{2}$, MAXIM \\ RUBIN-BLUM ${ }^{3}$, MICHAL ELUL $^{4}$, MARCUS ELVERT ${ }^{5}$ AND \\ ORIT SIVAN $^{6}$ \\ ${ }^{1}$ Ben-Gurion University of the Negev \\ ${ }^{2}$ Israel Oceanographic and Limnoilogical Research, The Yigal \\ Allon Kinneret Limnological Laboratory \\ ${ }^{3}$ Israel Limnology and Oceanography Research \\ ${ }^{4}$ Ben Gurion university un the negev, Israel \\ ${ }^{5}$ MARUM - Center for Marine Environmental Sciences \\ ${ }^{6}$ Ben Gurion University of the Negev \\ Presenting Author: hannil@post.bgu.ac.il
}

\begin{abstract}
Methane is a potent greenhouse gas that is produced naturally via microbial processes in anoxic environments (i.e. marine and lake sediments). Anaerobic oxidation of methane (AOM) is one of the major processes that limit the release of the greenhouse gas methane from natural environments. AOM is driven by a variety of electron acceptors, including sulfate, iron, manganese, nitrate, nitrite and organic electron shuttles such as humic substances. Iron-coupled AOM (Fe-AOM) was suggested to play a role in the oxidation of methane in Lake Kinneret methanic fresh sediments by anaerobic methane-oxidizers (ANME) or methanogens, combined with aerobic methanotrophy. Nevertheless, the mechanism and the way they collaborate, if at all, is still unknown. Here we performed long-term incubations of Lake Kinneret methanic sediments with ${ }^{13} \mathrm{C}$-labeled methane and various electron acceptors and inhibitors, to investigate how methane oxidation is performed. Geochemical measurements indicate, considerable methane oxidation (up to $2 \mu \mathrm{M} \mathrm{d}^{-1}$ ) in a net methanogenesis environment, and a shift in electron acceptor utilization: from anaerobic and aerobic methane oxidation in fresh incubated sediments to strictly anaerobic methane oxidation in pre-incubated sediment experiments. Iron oxides and humic substances might support this AOM, while sulfate, nitrate and nitrite do not. Additional organic geochemical and metagenomic analyses suggest that the AOM in the long-term incubations is performed by methanogens through reverse methanogenesis either metabolically or by back flux reactions.
\end{abstract}

